\title{
IMPACTOS DE SISTEMAS AGROFLORESTAIS E CONVENCIONAL SOBRE A QUALIDADE DO SOLO NO SEMI-ÁRIDO CEARENSE ${ }^{1}$
}

\author{
Stoécio Malta Ferreira Maia², Francisco Alisson da Silva Xavier², Teógenes Senna de Oliveira² ${ }^{2}$ Eduardo \\ de Sá Mendonça ${ }^{3}$ e João Ambrósio de Araújo Filho ${ }^{4}$
}

\begin{abstract}
RESUMO - Os benefícios gerados pelos sistemas agroflorestais (SAFs) ao ambiente são comprovados nas mais diversas regiões do mundo. No entanto, na região semi-árida cearense, são poucos os trabalhos desenvolvidos englobando as vantagens atribuídas a este tipo de sistema quanto ao uso do solo. Objetivou-se avaliar o impacto de quatro SAFs e um convencional sobre a qualidade do solo, comparativamente à condição natural (caatinga nativa), após cinco anos de uso na região semi-árida cearense. Os tratamentos testados foram: agrossilvipastoril (AGP); silvipastoril (SILV); tradicional cultivado em 1998 e 1999 (TR98); tradicional cultivado em 2002 (TR02); e cultivo intensivo (CI) e duas áreas de mata nativa (MN-1 e MN-2), que foram usadas como referência de um estado de equilíbrio. O impacto dos sistemas de manejo sobre a qualidade do solo foi medido pelas seguintes variáveis: a granulometria; argila dispersa; a estabilidade e distribuição porcentual dos agregados; os teores de $\mathrm{Ca}^{2+}, \mathrm{Mg}^{2+}, \mathrm{K}^{+}, \mathrm{Na}^{+}$trocáveis; a acidez potencial; o pH do solo; e o teor de carbono orgânico total (COT), considerando-se as profundidades de 0-6, 6-12, 12-20 e 20-40 cm. Os tratamentos AGP, TR98 e CI promoveram maior revolvimento do solo provocando a redução nos teores de COT. Os atributos químicos, como bases trocáveis, capacidade de troca catiônica, $\mathrm{pH}$ e acidez potencial nos tratamentos SILV e MN-2, variaram em função dos teores de argila, enquanto nos tratamentos TR98 e CI a redução nesses atributos foi devido ao maior revolvimento do solo. O tratamento AGP mostrou-se eficiente na ciclagem de nutrientes, entretanto, o revolvimento do solo e a concomitante redução do teor de COT, geraram também diminuição na estabilidade de agregados. Os resultados obtidos permitem recomendar o tratamento SILV para a manutenção da qualidade do solo e produção de alimentos na região do semi-árido cearense.
\end{abstract}

Palavras-chave: Matéria orgânica, semi-árido, ciclagem de nutrientes e qualidade do solo.

\section{THE IMPACT OF AGROFORESTRY AND CONVENTIONAL SYSTEMS ON THE SOIL QUALITY FROM CEARENSE SEMI-ARID REGION}

\begin{abstract}
The environmental benefits of agroforestry system are well documented in many regions of the globe. However, works documenting the advantage of this kind of soil management system on soil quality in the cearense semi-arid region are very scarce. The aim of this work was to evaluate the impact of agroforestry and conventional systems on the physical and chemical soil characteristics, after five years of experimentation in the semi-arid region, Ceará, Brazil. The experiment consisted of seven treatments: agrosilvopasture (AGP), silvopasture (SILV), traditionally cultivated in 1998 and 1999 (TR98), traditionally cultivated in 2002 (TRO2), intensive cropping (CI), and two native forests (MN-1 and $M N-2)$. Soil samples were collected at four depths: $0-6,6-12,12-20$ and 20-40cm. Soil quality was evaluated by the following physical and chemical characteristics analyzed: granulometry, clay dispersion, aggregate stability, exchangeable elements, soil pH and total organic carbon (COT). The treatments AGP, TR98 and CI reduced the COT content due to intensive soil management.
\end{abstract}

\footnotetext{
${ }^{1}$ Recebido em 21.12.2004 e aceito para publicação em 05.04.2006.

${ }^{2}$ Departamento de Ciências do Solo da Universidade Federal do Ceará - UFC. Campus do PICI, Bloco 807, 60455-760 Fortaleza-CE. E-mail: <teo@ufc.br>.

${ }^{3}$ Departamento de Solos da Universidade Federal de Viçosa -UFV. 36570-000 Viçosa-MG. E-mail: <esm@ufv.br>.

${ }^{4}$ Embrapa Caprinos. Estrada Sobral-Groaíras, Km 4-Cx. Postal D-10.62011-970 Sobral-CE. E-mail:<ambrosio@cnpc.embrapa.br>.
} 


\begin{abstract}
Chemical characteristics, such as exchangeable cations, CEC, and pH varied as a function of clay content in SILV and MN-2, whereas in CI and TR98, there was reduction in these characteristics due to the intensive soil management. The AGP treatment seems to improve the nutrient cycle, however, the intensive soil management, and the consequent reduction in COT content also originated reduction in aggregate stability. Results allowed to recommend the SILV treatment in order to maintain soil quality and food production in the cearense semiarid region.
\end{abstract}

Keywords: Organic matter, semi arid, nutrient cycling and soil quality.

\section{INTRODUÇÃO}

As regiões semi-áridas são caracterizadas por terem altas temperaturas, reduzida pluviosidade, solos pouco intemperizados e pequena produção de fitomassa. Somando-se a essas condições edafoclimáticas, observase, no semi-árido brasileiro, a adoção de sistemas agrícolas totalmente extrativistas. Na pecuária, o que sobressai é o superpastoreio. A agricultura é desenvolvida à custa de um desmatamento indiscriminado, queimadas e períodos de pousio inadequados. Há ainda intensa extração de lenha e madeira para atender à demanda familiar, de cerâmicas e padarias, contribuindo enormemente para a redução da vegetação da caatinga (ARAÚJO FILHO, 2002).

Como resultado de todos esses fatores, a região semi-árida no Nordeste brasileiro configura-se em um ambiente extremamente frágil, tanto do ponto de vista ambiental quanto socioeconômico (BREMAN e KESSLER, 1997; SÁNCHEZ, 2001).

Nesse contexto, opções racionais de uso do solo devem ser propostas e estudadas. Sistemas agroflorestais (SAFs) têm sido amplamente promovidos como sistemas de produção agrícola sustentáveis e particularmente atraentes para regiões subdesenvolvidas, onde o uso de insumos externos é inviável (BREMAN e KESSLER, 1997).

Os SAFs apresentam inúmeras vantagens que contribuem para o estabelecimento de modelos de produção mais estáveis e que podem amenizar as adversidades encontradas pela agropecuária nas regiões semi-áridas. Esses sistemas proporcionam maior cobertura do solo, favorecem a preservação da fauna e da flora, promovem a ciclagem de nutrientes a partir da ação de sistemas radiculares diversos e propiciam um contínuo aporte de matéria orgânica (BREMAN e KESSLER, 1997; ARAÚJO et al., 2001; SÁNCHEZ, 2001; SCHROTH et al., 2002). Possibilitam, ainda, maior diversidade de produtos a serem explorados, o que alivia a sazonalidade, fenômeno comum no setor agropecuário (IZAC e SANCHEZ, 2001).

Apesar das vantagens anteriormente relacionadas e de o conceito de SAFs preconizar o manejo conservacionista do solo como pré-requisito básico de sua adoção (NAIR, 1993), são praticamente inexistentes na região semi-árida do Brasil pesquisas voltadas para a avaliação da potencialidade dos SAFs para a produção de alimentos e melhoria da qualidade do solo. Portanto, o conhecimento das alterações em condições específicas de solo e clima é fundamental para o entendimento da potencialidade desses sistemas em relação à produtividade das culturas e na adoção de práticas no sentido de contornar possíveis limitações advindas da sua utilização (BAYER e MIELNICZUK, 1997).

Considerando os aspectos apresentados, o presente estudo objetivou avaliar o impacto de quatro SAFs e um convencional sobre a qualidade do solo, comparativamente à condição natural (caatinga nativa), após cinco anos de uso na região semi-árida cearense, em experimento instalado no Município de Sobral, CE.

\section{MATERIAL E MÉTODOS}

\subsection{Localização e descrição das áreas}

Este estudo foi realizado na Fazenda Crioula, pertencente ao Centro Nacional de Pesquisa de Caprinos (CNPC)/EMBRAPA, situada no Município de Sobral, CE. O município encontra-se na região semi-árida cearense e está a $3^{\circ} 41^{\prime} \mathrm{S}$ e $40^{\circ} 20^{\prime} \mathrm{W}$, com altitude de $69 \mathrm{~m}$. As médias anuais de temperatura e precipitação são de $30^{\circ} \mathrm{C}$ e $798 \mathrm{~mm}$, respectivamente. O solo dominante é um Luvissolo Crômico Órtico. 
Os sistemas avaliados foram escolhidos a partir de projeto de pesquisa desenvolvido no CNPC desde 1997, que tem como objetivo avaliar três sistemas agrossilvipastoris de produção: o tradicional, o agrossilvipastoril fixo e o agrossilvipastoril rotativo. Além desses, avaliou-se, também, um sistema de cultivo convencional. Nesse contexto, foram selecionados e estudados os seguintes tratamentos: Tratamento Agrossilvipastoril (AGP): oriundo do modelo rotativo, que adotou o cultivo em aléias, com faixas de 3,0 m de largura, cultivando-se milho intercalado por fileiras de leucena, implantadas com espaçamento de $0,5 \mathrm{~m}$ entre plantas. No período seco, a parcela foi utilizada como banco de proteína, onde o rebanho de 20 matrizes ovinas permanecia por uma hora diariamente, pela manhã. Tratamento Silvipastoril (SILV): esse tratamento é também oriundo do modelo rotativo e consistiu de uma área de 4,8 ha que foi submetida ao processo de raleamento, preservando-se aproximadamente 260 árvores por hectare, o que correspondeu a $38 \%$ de cobertura. Essa área serviu, juntamente com a área de mata nativa-1, como pastagem de mantença do rebanho de 20 matrizes ovinas. Tratamento Agrossilvipastoril Tradicional - 1998 (TR98): esse tratamento é proveniente do sistema tradicional, em que uma área de 0,8 ha foi submetida ao desmatamento que produziu 16,2 e 12,7 $\mathrm{Mg} \mathrm{ha}^{-1}(\mathrm{MS})$ de restolhos lenhoso e folhoso, respectivamente, que foram queimados no ano de 1997. A parcela foi cultivada com milho em 1998 e 1999 e, em seguida, deixada em pousio. No período seco, a forragem e os resíduos culturais da área cultivada foram usados para a suplementação alimentar de um rebanho de 10 matrizes ovinas. Tratamento Agrossilvipastoril Tradicional - 2002 (TR02): área proveniente do sistema tradicional, que foi submetida ao desmatamento e queima da vegetação lenhosa em 2001, sendo cultivada com milho em 2002, ano em que foi realizada a coleta de solo. No período seco, a forragem e os resíduos culturais da área cultivada foram usados para a suplementação alimentar de um rebanho de 10 matrizes ovinas. Cultivo intensivo (CI): esse tratamento corresponde ao sistema convencional, em que uma parcela de 1,0 ha foi desmatada e queimada em 1997 e cultivada com milho de 1998 a 2002 . Mata nativa -1 (MN-1): área de caatinga nativa do sistema rotativo sendo considerada como testemunha, utilizada no período seco como área de mantença dos animais. Mata nativa -2 (MN-2): área de caatinga nativa sendo considerada como testemunha, sobretudo para o tratamento CI, utilizada no período seco como área de mantença dos animais.

\subsection{Amostragem e atributos físicos e químicos}

Em setembro de 2002, cinco perfis foram abertos em cada tratamento, considerando-se como repetições. As amostras de solo foram coletadas em quatro profundidades: 0-6, 6-12, 12-20 e 20-40 cm.

Utilizou-se o método da pipeta para as determinações dos teores de argila total e argila dispersa, com e sem utilização do dispersante $\mathrm{NaOH} 0,1 \mathrm{~mol} \mathrm{~L}^{-1}$, respectivamente, segundo Day (1965). As frações areia grossa e areia fina foram obtidas por peneiramento e o silte, por diferença.

A estabilidade e distribuição porcentual dos agregados do solo foi medida utilizando-se o método da tamisação em água, separando agregados nas seguintes classes: C1 (4,76-2,0 mm), C2 (2,0-1,0 mm), C3 (1,0-0,50 mm), C4 (0,50-0,25 mm) e C5 (<0,25 mm) (EMBRAPA, 1997).

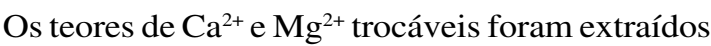
com $\mathrm{KCl} 1,0 \mathrm{~mol} \mathrm{~L}^{-1}$ e determinados por espectrofotometria de absorção atômica, enquanto os de $\mathrm{Na}^{+}$e $\mathrm{K}^{+}$trocáveis foram extraídos com Mehlich 1, sendo determinados em fotômetro de chama. Foram determinados o pH em água $(1: 2,5)$ e em $\mathrm{KCl}$ por potenciometria. A acidez potencial $(\mathrm{H}+\mathrm{Al})$ foi extraída com $\mathrm{Ca}(\mathrm{OAc})_{2} 0,5 \mathrm{~mol}$ $\mathrm{L}^{-1}$ e quantificada por titulometria com $\mathrm{NaOH} 0,0606$ mol L-1 (EMBRAPA, 1997). ACTC do solo foi estimada pela soma dos teores de $\mathrm{Ca}^{2+}, \mathrm{Mg}^{2+}, \mathrm{K}^{+}, \mathrm{Na}^{+}$trocáveis e $\mathrm{H}+\mathrm{Al}$. Os resultados foram expressos na relação massa-volume.

O carbono orgânico total do solo (COT) foi quantificado por oxidação da matéria orgânica via úmida, empregando-se solução de dicromato de potássio em meio sulfúrico, com fonte externa de calor (YEOMANS e BREMNER, 1988).

\subsection{Análise estatística}

O delineamento experimental utilizado foi em blocos ao acaso, com parcelas subdivididas no espaço. Foram considerados como efeitos primários e secundários os sistemas de manejo e as profundidades de coleta de solo, respectivamente. Os dados foram submetidos à análise de variância e teste de comparação de médias pelo teste de Tukey $(\mathrm{P}<0,05)$, utilizando-se o programa SAEG desenvolvido pela Universidade Federal de Viçosa.

R. Árvore, Viçosa-MG, v.30, n.5, p.837-848, 2006 


\section{RESULTADOS E DISCUSSÃO}

\subsection{Atributos físicos}

Os resultados obtidos para a granulometria foram variáveis e não apresentaram comportamento que pudesse ser atribuído ao manejo realizado nos tratamentos (Tabela 1). Desse modo, as diferenças observadas devem estar relacionadas, principalmente, à variabilidade espacial da área experimental. Segundo Klepker e Anghinoni (1995), alterações entre as frações granulométricas são difíceis de ocorrer e, quando ocorrem, são detectadas após períodos variados de uso.
Não houve diferenças entre os tratamentos quanto aos teores das frações areia grossa e fina do solo (Tabela 1). Entretanto, os tratamentos SILV e MN-2 apresentaram os maiores resultados para a fração areia grossa em todas as camadas. Quanto aos teores de silte, a área MN-2 apresentou menor teor quando comparada com os demais tratamentos, diferindo do AGP nas camadas de 0-6 e 6-12 cm e do TR02 apenas na camada superficial.

Com relação à fração argila, apenas o tratamento MN-2 (111,4 $\left.\mathrm{g} \mathrm{dm}^{-3}\right)$ diferiu do TR02 (360,5 $\left.\mathrm{g} \mathrm{dm}^{-3}\right)$, na camada de $6-12 \mathrm{~cm}$. Na camada de $12-20 \mathrm{~cm}$, os tratamentos SILV e MN-2 apresentaram os menores teores, diferindo apenas do TR02.

Tabela 1 - Médias dos teores de areia grossa, areia fina, silte e argila, nos diferentes tratamentos agroflorestais e convencional e em áreas nativas nas profundidades de 0-6, 6-12,12-20 e $20-40 \mathrm{~cm}$

Table 1 - Sandy, silt and clay contents from agroforestry, conventional and native forest sites at the 0-6, 6-12, 12-20 and $20-40 \mathrm{~cm}$ layers

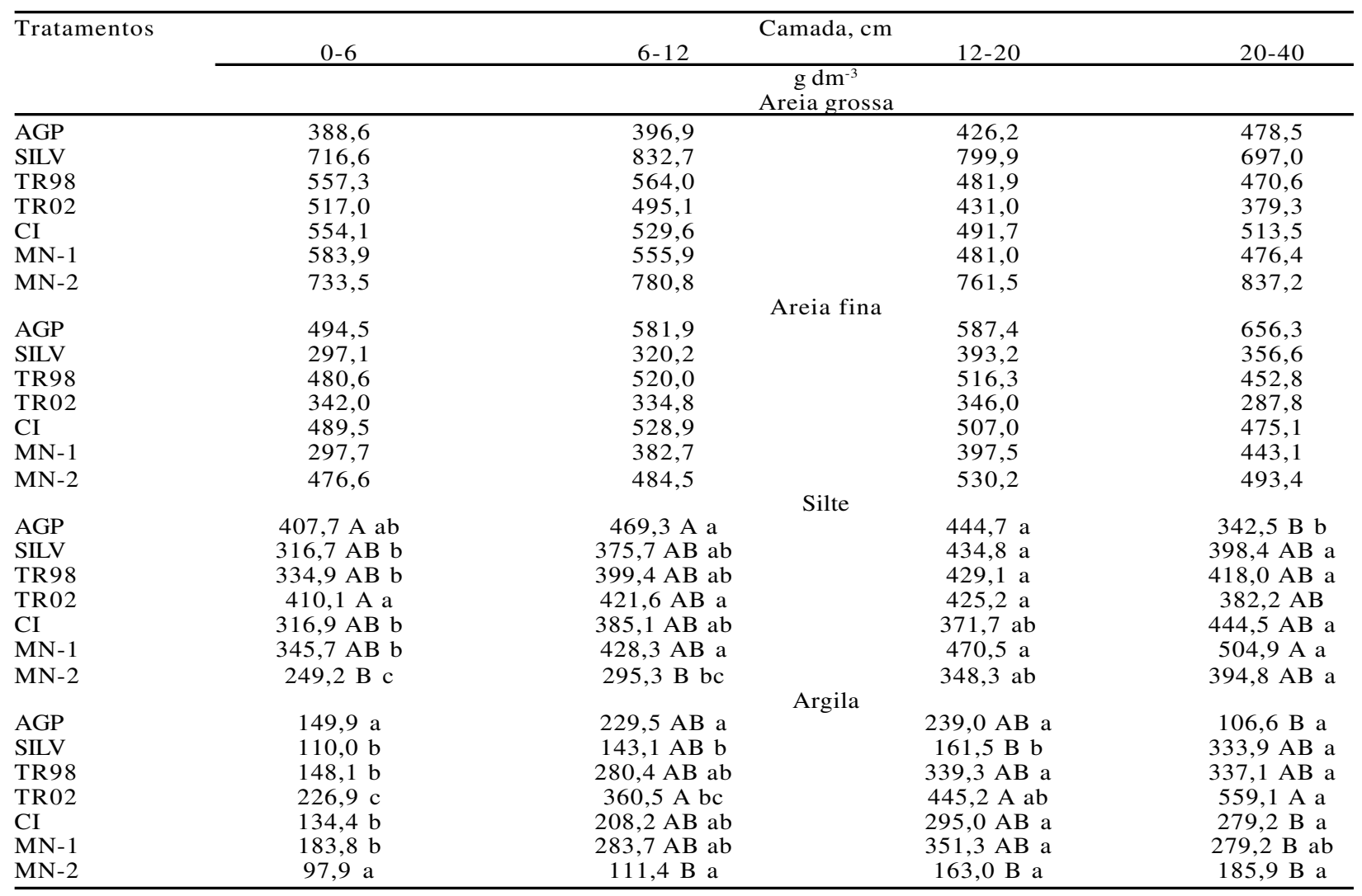

Médias seguidas de mesmas letras maiúsculas nas colunas e minúsculas nas linhas não diferem entre si, pelo teste de Tukey a 5\%. AGP: agrossilvipastoril; SILV: silvipastoril; TR98: tradicional de 1998; TR02: tradicional de 2002; CI: cultivo intensivo; MN-1: mata nativa 1; e MN-2: mata nativa 2.

Means followed by the same letter, capitals in the columns and small in the lines, are not significantly different by the Tukey test at 5\% level. AGP: agrosilvopasture; SILV: silvopasture; TR98: traditionally cultivated in 1998; TR02: traditionally cultivated in 2002; CI: intensive cropping; $M N-1$ : native forest $1 ; M N-2$ : native forest 2.

R. Árvore, Viçosa-MG, v.30, n.5, p.837-848, 2006 
Os teores de argila dispersa em água (ADA) (Tabela 2) apresentaram baixos valores quando comparados com outros estudos (OLIVEIRA et al., 1983; PALMEIRA et al., 1999), o que indica um bom estado de floculação nos solos dos tratamentos testados. De modo geral, houve incremento da ADA com a profundidade, percebendo-se a existência de uma relação direta entre o aumento da ADA e a diminuição do COT, concomitantemente ao aumento do teor de argila total. Os coeficientes de correlação de -0,337 e 0,911 entre o teor de COT e argila total, respectivamente, e a ADA corroboram essa assertiva.

\subsection{Atributos químicos}

Os tratamentos SILV e MN-2 apresentaram os menores teores de bases trocáveis no solo (Tabelas 3 e 4). Considerando que esses dois tratamentos receberam um aporte contínuo de matéria orgânica e não promoveram revolvimento do solo, possivelmente os menores teores de bases trocáveis estão associados às variações no conteúdo de argila nessas áreas, proporcionando aumento nos teores da fração areia, o que leva à redução dos sítios de troca, acarretando menor retenção dos elementos trocáveis. Foram encontrados coeficientes de correlação negativos de $-0,63,-0,47,-0,31$ e $-0,66$ entre os teores de $\mathrm{Ca}^{2+}, \mathrm{Mg}^{2+}, \mathrm{K}^{+}$e $\mathrm{Na}^{+}$trocáveis, respectivamente, e a fração areia grossa.

Os maiores teores de bases trocáveis foram obtidos no tratamento TR02. Contudo, esses resultados podem ser atribuídos à herança da vegetação nativa que o antecedeu, como também à queimada a que foi submetido meses antes da amostragem da área. Embora a queima dos resíduos vegetais possa elevar os teores das bases trocáveis, esta poderá, em longo prazo, diminuir a fertilidade do solo, uma vez que as cinzas são facilmente carreadas por lixiviação e, ou, erosão (MENDONZA et al., 2000). Os resultados encontrados no tratamento TR98 (similar a TR02) suportam essa hipótese, pois houve reduções de 30,1; 37,$1 ; 7,4$; e 13,0\% nos teores de $\mathrm{Ca}^{2+}, \mathrm{Mg}^{2+}, \mathrm{K}^{+} \mathrm{e} \mathrm{Na}^{+}$ trocáveis, respectivamente, quando relacionados ao tratamento $\mathrm{MN}-1$.

Os tratamentos AGP e CI, apesar de terem sido cultivados no mesmo período (cinco anos), apresentaram resultados divergentes quanto aos teores de bases trocáveis. O tratamento AGP manteve seus teores similares ao tratamento-referência $\mathrm{MN}$-1, enquanto em CI ocorreram perdas significativas (Tabelas 3 e 4). Esses resultados podem ser atribuídos ao contínuo aporte de resíduos orgânicos no tratamento AGP, como conseqüência da ação de sistemas radiculares diversificados, indicando maior eficiência na ciclagem de nutrientes. Vários trabalhos têm evidenciado o potencial dos SAFs, sobretudo daqueles que utilizam o cultivo em aléias na ciclagem de nutrientes (BREMAN e KESSLER, 1997; ROOSE e NDAYIZIGIYE, 1997; NAIR et al., 1999; FRANZEL et al., 2001).

Tabela 2 - Porcentagem de argila dispersa em água nos diferentes tratamentos agroflorestal e convencional e em áreas nativas, nas profundidades de $0-6,6-12,12-20$ e $20-40 \mathrm{~cm}$

Table 2 - Percentage of water-dispersed clay from agroforestry, conventional and native forest sites at the 0-6, 6-12, 12 20 and $20-40 \mathrm{~cm}$ layer

\begin{tabular}{|c|c|c|c|c|}
\hline \multirow[t]{2}{*}{ Tratamentos } & \multicolumn{4}{|c|}{ Camada, $\mathrm{cm}$} \\
\hline & $0-6$ & $6-12$ & $12-20$ & $20-40$ \\
\hline & \multicolumn{4}{|c|}{$\%$} \\
\hline$\overline{A G P}$ & $4,66 \mathrm{a}$ & $7,17 \mathrm{AB}$ a & $6,61 \mathrm{BC} \mathrm{a}$ & $6,22 \mathrm{~B} \mathrm{a}$ \\
\hline SILV & $3,47 \mathrm{~b}$ & $5,26 \mathrm{AB} \mathrm{b}$ & $6,0 \mathrm{BC} \mathrm{b}$ & $9,33 \mathrm{~B}$ a \\
\hline TR98 & $4,35 \mathrm{c}$ & $5,77 \mathrm{AB} b \mathrm{c}$ & $7,36 \mathrm{BC} \mathrm{ab}$ & $9,33 \mathrm{~B}$ a \\
\hline TR02 & $4,26 \mathrm{c}$ & $8,93 \mathrm{~A} \mathrm{~b}$ & $11,7 \mathrm{~A} \mathrm{ab}$ & $14,15 \mathrm{~A} \mathrm{a}$ \\
\hline CI & $3,88 \mathrm{c}$ & $5,57 \mathrm{AB} b \mathrm{c}$ & $7,31 \mathrm{BC} \mathrm{ab}$ & $9,62 \mathrm{~B}$ a \\
\hline MN-1 & $4,27 \mathrm{~b}$ & $6,82 \mathrm{AB} b$ & $9,94 \mathrm{AB}$ a & $10,2 \mathrm{AB}$ a \\
\hline MN-2 & $2,42 \mathrm{~b}$ & $4,12 \mathrm{~B} \mathrm{ab}$ & $4,25 \mathrm{C} \mathrm{ab}$ & $5,94 \mathrm{~B} \mathrm{a}$ \\
\hline
\end{tabular}

Médias seguidas de mesmas letras maiúsculas nas colunas e minúsculas nas linhas não diferem entre si pelo teste de Tukey a 5\%. AGP: agrossilvipastoril; e SILV: silvipastoril; TR98: tradicional de 1998; TR02: tradicional de 2002; CI: cultivo intensivo; MN-1: mata nativa 1 ; MN-2: mata nativa 2.

Means followed by the same letter, capitals in the columns and small in the lines, are not significantly different by the Tukey test at 5\% level. AGP: agrosilvopasture; SILV: silvopasture; TR98: traditionally cultivated in 1998; TR02: traditionally cultivated in 2002; CI: intensive cropping; $M N-1$ : native forest $1 ; M N-2$ : native forest 2. 
Tabela 3 - Médias dos teores de $\mathrm{Ca}^{2+}$ e $\mathrm{Mg}^{2+}$ trocáveis nos diferentes tratamentos agroflorestal e convencional e em áreas nativas, nas profundidades de 0-6, 6-12, 12-20 e 20-40 cm

Table 3 - Exchangeable soil $\mathrm{Ca}^{2+}$ and $\mathrm{Mg}^{2+}$ contents from agroforestry, conventional and native forest sites at the 0-6, 6-12, $12-20$ and $20-40 \mathrm{~cm}$ layers

\begin{tabular}{|c|c|c|c|c|c|c|c|c|}
\hline \multirow[t]{2}{*}{ Tratamentos } & & \multicolumn{7}{|c|}{ Camada, $\mathrm{cm}$} \\
\hline & $0-6$ & $6-12$ & $12-20$ & $20-40$ & $0-6$ & $6-12$ & $12-20$ & $20-40$ \\
\hline & \multicolumn{4}{|c|}{$\mathrm{Ca}^{2+}, \mathrm{cmol}_{\mathrm{c}} \mathrm{dm}^{-3}$} & \multicolumn{4}{|c|}{$\mathrm{Mg}^{2+}, \mathrm{cmol}_{\mathrm{c}} \mathrm{dm}^{-3}$} \\
\hline AGP & $20,8 \mathrm{BCa}$ & $21,9 \mathrm{Aa}$ & $22,8 \mathrm{Aa}$ & $21,8 \mathrm{Aa}$ & 4,5 & 4,0 & 4,5 & 4,3 \\
\hline SILV & $11,3 \mathrm{BCa}$ & $6,4 \mathrm{Cab}$ & $4,2 \mathrm{Cab}$ & $3,2 \mathrm{Bb}$ & 2,7 & 2,5 & 3,5 & 3,4 \\
\hline TR98 & $15,4 \mathrm{BCa}$ & $16,8 \mathrm{ABC}$ a & $16,1 \mathrm{ABa}$ & 19,2 Аа & 4,2 & 5,2 & 7,3 & 5,4 \\
\hline TR02 & $31,9 \mathrm{Aa}$ & $17,7 \mathrm{ABb}$ & $17,9 \mathrm{ABb}$ & $15,7 \mathrm{Ab}$ & 4,6 & 8,2 & 9,3 & 9,0 \\
\hline CI & $13,5 \mathrm{BCa}$ & $13,8 \mathrm{ABCa}$ & $14,9 \mathrm{ABC}$ a & $15,4 \mathrm{Aa}$ & 2,8 & 2,9 & 4,0 & 4,9 \\
\hline $\mathrm{MN}-1$ & $22,0 \mathrm{ABa}$ & $22,2 \mathrm{Aa}$ & $22,5 \mathrm{Aa}$ & $21,6 \mathrm{Aa}$ & 6,7 & 7,7 & 10,7 & 13,5 \\
\hline $\mathrm{MN}-2$ & $10,8 \mathrm{Ca}$ & $8,0 \mathrm{BCa}$ & $9,4 \mathrm{BCa}$ & $12,6 \mathrm{ABa}$ & 2,9 & 2,9 & 3,1 & 4,2 \\
\hline
\end{tabular}

Médias seguidas de mesmas letras maiúsculas nas colunas e minúsculas nas linhas, não diferem entre si, pelo teste de Tukey a 5\%. AGP: agrossilvipastoril; SILV: silvipastoril; TR98: tradicional de 1998; TR02: tradicional de 2002; CI: cultivo intensivo; MN-1: mata nativa 1 ; e MN-2: mata nativa 2.

Means followed by the same letter, capitals in the columns and small in the lines, are not significantly different by the Tukey test at 5\% level. AGP: agrosilvopasture; SILV: silvopasture; TR98: traditionally cultivated in 1998; TRO2: traditionally cultivated in 2002; CI: intensive cropping; $M N-1$ : native forest $1 ; M N-2$ : native forest 2.

Tabela 4 - Médias dos teores de $\mathrm{K}^{+}$e $\mathrm{Na}^{+}$trocáveis nos diferentes tratamentos agroflorestal e convencional e em áreas nativas, nas profundidades de 0-6, 6-12,12-20 e 20-40 cm

Table 4 - Exchangeable soil $K$ and Na contents from agroforestry, conventional and native forest sites at the 0-6, 6-12, $12-20$ and $20-40 \mathrm{~cm}$ layers

\begin{tabular}{|c|c|c|c|c|c|c|c|c|}
\hline \multirow[t]{2}{*}{ Tratamentos } & \multicolumn{8}{|c|}{ Camada, $\mathrm{cm}$} \\
\hline & $0-6$ & $6-12$ & $12-20$ & $20-40$ & $0-6$ & $6-12$ & $12-20$ & $20-40$ \\
\hline & \multicolumn{5}{|c|}{$\mathrm{K}^{+}, \mathrm{cmol}_{\mathrm{c}} \mathrm{dm}^{-3}$} & \multicolumn{3}{|c|}{$\mathrm{Na}^{+}, \mathrm{cmol}_{\mathrm{c}} \mathrm{dm}^{-3}$} \\
\hline AGP & $1,57 \mathrm{Aa}$ & $0,91 \mathrm{ABb}$ & $0,65 \mathrm{Bbc}$ & $0,28 \mathrm{c}$ & $0,19 \mathrm{ABa}$ & $0,19 \mathrm{Aa}$ & $0,22 \mathrm{Aa}$ & $0,20 \mathrm{Aa}$ \\
\hline SILV & $1,07 \mathrm{ABa}$ & $0,73 \mathrm{Ba}$ & $0,71 \mathrm{Ba}$ & $0,91 \mathrm{a}$ & $0,10 \mathrm{Ba}$ & $0,08 \mathrm{Ba}$ & $0,06 \mathrm{Ba}$ & $0,07 \mathrm{Ba}$ \\
\hline TR98 & $1,38 \mathrm{ABa}$ & $1,56 \mathrm{Aa}$ & $1,25 \mathrm{ABa}$ & $0,67 \mathrm{~b}$ & $0,14 \mathrm{ABb}$ & $0,17 \mathrm{ABab}$ & $0,19 \mathrm{Aab}$ & $0,24 \mathrm{Aa}$ \\
\hline TR02 & $1,75 \mathrm{Aa}$ & $1,22 \mathrm{ABab}$ & $0,88 \mathrm{ABbc}$ & $0,58 \mathrm{c}$ & $0,22 \mathrm{Aa}$ & $0,16 \mathrm{ABab}$ & $0,15 \mathrm{ABab}$ & $0,15 \mathrm{Abb}$ \\
\hline CI & $1,04 \mathrm{ABa}$ & $0,67 \mathrm{Bab}$ & $0,59 \mathrm{Bab}$ & $0,38 \mathrm{~b}$ & $0,12 \mathrm{Abb}$ & $0,13 \mathrm{Abb}$ & $0,15 \mathrm{AB} a b$ & $0,21 \mathrm{Aa}$ \\
\hline MN-1 & 1,49 Aab & $1,07 \mathrm{ABab}$ & $1,59 \mathrm{Aa}$ & $0,95 \mathrm{~b}$ & $0,17 \mathrm{ABa}$ & $0,18 \mathrm{ABa}$ & $0,22 \mathrm{Aa}$ & $0,22 \mathrm{Aa}$ \\
\hline MN-2 & $0,60 \mathrm{Ba}$ & $0,53 \mathrm{Ba}$ & $0,49 \mathrm{Ba}$ & $0,46 \mathrm{a}$ & $0,09 \mathrm{Ba}$ & $0,12 \mathrm{ABa}$ & $0,12 \mathrm{ABa}$ & $0,16 \mathrm{ABa}$ \\
\hline
\end{tabular}

Médias seguidas de mesmas letras maiúsculas nas colunas e minúsculas nas linhas não diferem entre si pelo teste de Tukey a 5\%. AGP: agrossilvipastoril; SILV: silvipastoril; TR98: tradicional de 1998; TR02: tradicional de 2002; CI: cultivo intensivo; MN-1: mata nativa 1; e MN-2: mata nativa 2 .

Means followed by the same letter, capitals in the columns and small in the lines, are not significantly different by the Tukey test at 5\% level. AGP: agrosilvopasture; SILV: silvopasture; TR98: traditionally cultivated in 1998; TR02: traditionally cultivated in 2002; CI: intensive cropping; $M N-1$ : native forest $1 ; M N-2$ : native forest 2.

Os resultados obtidos para a capacidade de troca catiônica (CTC) do solo e para a saturação por bases (V) nos tratamentos (Tabela 5) refletiram o comportamento das bases trocáveis do solo e se mostraram elevados quando comparados com os resultados de Luz et al. (1992) em um Luvissolo no semi-árido do Estado de Pernambuco. Os tratamentos TR02, MN-1 e AGP apresentaram os maiores valores de CTC, enquanto os tratamentos SILV seguido de MN-2 apresentaram os menores resultados. Em profundidade, a CTC manteve- se constante em decorrência do aumento do teor de argila. Quanto à saturação por bases, observou-se que o tratamento AGP exibiu os maiores valores em todas as profundidades, confirmando a eficiência desse sistema na ciclagem de nutrientes.

$\mathrm{O}$ pH em água variou de levemente ácido a levemente alcalino (Tabela 5), estando sempre em uma faixa ótima para o desenvolvimento das culturas (MELLO et al., 1983). O pH em KCl (Tabela 5) apresentou comportamento similar ao $\mathrm{pH}$ em água, e os seus valores 
até a segunda camada, geralmente maiores que 5,5, indicaram que o $\mathrm{Al}^{3+}$ tem pouca participação na acidez do solo. $\mathrm{Na}$ terceira e, principalmente, na quarta camada, $\mathrm{o} \mathrm{Al}^{3+}$ pode estar aumentando sua participação na acidez potencial, pois nessas camadas ocorreram valores de $\mathrm{pH}$ abaixo de 5,5, o que provocaria a solubilização do alumínio para a solução do solo (MELLO et al., 1983; TAN, 1998).

Os resultados de $\Delta \mathrm{pH}$ (Tabela 5) indicam a predominância de cargas negativas nos solos avaliados. Dessa forma, quanto maior o $\Delta \mathrm{pH}$, maior a carga líquida negativa, implicando maior capacidade de adsorção de cátions. Observou-se, portanto, que os tratamentos AGP e MN-1 apresentaram maiores valores de $\Delta \mathrm{pH}$ e altos valores de CTC. No entanto, deve-se considerar que alta carga líquida negativa pode provocar, em algumas situações, maior dispersão das argilas, devido à repulsão eletrostática, acarretando a desagregação das partículas do solo, o que contribui com a perda de sua qualidade física (THEODORO et al., 2003).

\subsection{Carbono orgânico total e aporte orgânico}

Os teores de COT nos tratamentos CI, AGP e TR98 foram reduzidos em 40,3, 38,4 e 35\%, respectivamente, em relação à área nativa $\mathrm{MN}-1$ na camada de 0-6 cm. Nas demais camadas, confirmou-se a tendência de os teores de COT serem menores nesses tratamentos (Tabela 6). Tais resultados estão condizentes com os obtidos por Solomon et al. (2000), que verificaram perdas de até 55,5\% nos teores de COT em umaárea cultivada convencionalmente em um Luvissolo Crômico, na região semi-árida da Tanzânia.

Tabela 5 - Médias dos valores da capacidade de troca catiônica (CTC) solo, saturação por bases (V), pH em água, pH em $\mathrm{KCl}, \Delta \mathrm{pH}$ e acidez potencial $(\mathrm{H}+\mathrm{Al})$, nos diferentes tratamentos agroflorestal e convencional e em áreas nativas, nas profundidades de $0-6,6-12,12-20$ e $20-40 \mathrm{~cm}$

Table 5 - Cation exchangeable capacity $(C T C)$, base saturation $(V)$, water and $K C l p H, \Delta p H$ and potential acidity $(H+A l)$ from agroforestry, conventional and native forest sites at the 0-6, 6-12,12-20 and 20-40 cm layers

\begin{tabular}{|c|c|c|c|c|c|c|c|c|}
\hline \multirow[t]{2}{*}{$\overline{\text { Tratamentos }}$} & \multicolumn{8}{|c|}{ Camada, $\mathrm{cm}$} \\
\hline & $0-6$ & $6-12$ & $12-20$ & $20-40$ & $0-6$ & $6-12$ & $12-20$ & $20-40$ \\
\hline & \multicolumn{4}{|c|}{ CTC, $\mathrm{cmol}_{\mathrm{c}} \mathrm{dm}^{-3}$} & \multicolumn{4}{|c|}{$\mathrm{V}, \%$} \\
\hline AGP & 27,36 & 27,82 & 29,39 & 27,54 & $98,7 \mathrm{~A}$ & $97,2 \mathrm{~A}$ & $96,1 \mathrm{~A}$ & $\overline{96,6 \mathrm{~A}}$ \\
\hline SILV & 17,42 & 11,72 & 11,00 & 10,69 & $86,7 \mathrm{~B}$ & $82,0 \mathrm{C}$ & $77,7 \mathrm{C}$ & $71,6 \mathrm{C}$ \\
\hline TR98 & 22,40 & 25,83 & 27,92 & 28,27 & $94,4 \mathrm{AB}$ & $92,3 \mathrm{AB}$ & $89,7 \mathrm{AB}$ & $90,1 \mathrm{AB}$ \\
\hline TR02 & 38,93 & 28,66 & 30,77 & 28,58 & $98,5 \mathrm{~A}$ & $95,3 \mathrm{~A}$ & $91,9 \mathrm{AB}$ & $89,4 \mathrm{AB}$ \\
\hline $\mathrm{CI}$ & 19,08 & 19,08 & 21,58 & 23,04 & $91,6 \mathrm{AB}$ & $91,5 \mathrm{ABC}$ & $91,0 \mathrm{AB}$ & $90,9 \mathrm{AB}$ \\
\hline MN-1 & 32,28 & 32,97 & 37,88 & 39,19 & $93,7 \mathrm{AB}$ & $94,1 \mathrm{~A}$ & $92,0 \mathrm{AB}$ & $92,3 \mathrm{AB}$ \\
\hline \multirow[t]{2}{*}{$\mathrm{MN}-2$} & 17,11 & 13,97 & 15,89 & 20,23 & $84,8 \mathrm{~B}$ & $83,4 \mathrm{BC}$ & $82,5 \mathrm{BC}$ & $86,2 \mathrm{~B}$ \\
\hline & \multicolumn{4}{|c|}{ pH em água } & \multicolumn{4}{|c|}{$\mathrm{pH} \mathrm{em} \mathrm{KCl}$} \\
\hline AGP & $7,26 \mathrm{AB}$ & $7,13 \mathrm{~A}$ & $6,9 \mathrm{~A}$ & $6,76 \mathrm{AB}$ & $6,4 \mathrm{AB}$ & $5,73 \mathrm{AB}$ & 5,26 & 4,63 \\
\hline SILV & $6,66 \mathrm{BC}$ & $6,43 \mathrm{BC}$ & $6,26 \mathrm{AB}$ & $6,16 \mathrm{BC}$ & $5,96 \mathrm{~B}$ & $5,46 \mathrm{AB}$ & 5,10 & 4,70 \\
\hline TR98 & $6,9 \mathrm{ABC}$ & $7,13 \mathrm{AB}$ & $6,66 \mathrm{~A}$ & $6,46 \mathrm{ABC}$ & $6,23 \mathrm{AB}$ & $5,60 \mathrm{AB}$ & 5,13 & 4,83 \\
\hline TR02 & $7,53 \mathrm{~A}$ & $7,06 \mathrm{AB}$ & $6,8 \mathrm{~A}$ & $6,46 \mathrm{ABC}$ & $6,90 \mathrm{~A}$ & $6,10 \mathrm{~A}$ & 5,43 & 5,03 \\
\hline $\mathrm{CI}$ & $6,76 \mathrm{BC}$ & $6,9 \mathrm{AB}$ & $6,83 \mathrm{~A}$ & $6,86 \mathrm{~A}$ & $6,00 \mathrm{~B}$ & $5,73 \mathrm{AB}$ & 5,50 & 5,13 \\
\hline $\mathrm{MN}-1$ & $7,0 \mathrm{ABC}$ & $6,83 \mathrm{AB}$ & $6,7 \mathrm{~A}$ & $6,5 \mathrm{ABC}$ & $6,16 \mathrm{AB}$ & $5,66 \mathrm{AB}$ & 5,23 & 4,70 \\
\hline \multirow[t]{2}{*}{$\mathrm{MN}-2$} & $6,36 \mathrm{C}$ & $6,16 \mathrm{C}$ & $6,0 \mathrm{~B}$ & $6,06 \mathrm{C}$ & $5,63 \mathrm{~B}$ & $5,16 \mathrm{~B}$ & 4,80 & 4,43 \\
\hline & \multicolumn{4}{|c|}{$\Delta \mathrm{pH}$} & \multicolumn{4}{|c|}{$\mathrm{H}+\mathrm{Al}, \mathrm{cmol}_{\mathrm{c}} \mathrm{dm}^{-3}$} \\
\hline AGP & $-0,86$ & $-1,40$ & $-1,64$ & $-2,13$ & 0,32 & 0,73 & 1,16 & 0,95 \\
\hline SILV & $-0,70$ & $-0,97$ & $-1,16$ & $-1,46$ & 2,27 & 2,02 & 2,47 & 3,04 \\
\hline TR98 & $-0,67$ & $-1,53$ & $-1,53$ & $-1,63$ & 1,24 & 2,06 & 3,06 & 2,68 \\
\hline TR02 & $-0,63$ & $-0,96$ & $-1,37$ & $-1,43$ & 0,38 & 1,34 & 2,43 & 3,02 \\
\hline CI & $-0,76$ & $-1,17$ & $-1,33$ & $-1,73$ & 1,56 & 1,54 & 1,91 & 2,03 \\
\hline MN-1 & $-0,84$ & $-1,17$ & $-1,47$ & $-1,80$ & 1,97 & 1,82 & 2,87 & 2,81 \\
\hline $\mathrm{MN}-2$ & $-0,73$ & $-1,00$ & $-1,20$ & $-1,63$ & 2,26 & 2,39 & 2,78 & 2,72 \\
\hline
\end{tabular}

Médias seguidas de mesmas letras maiúsculas nas colunas e minúsculas nas linhas não diferem entre si, pelo teste de Tukey a 5\%. AGP: agrossilvipastoril; SILV: silvipastoril; TR98: tradicional de 1998; TR02: tradicional de 2002; CI: cultivo intensivo; MN-1: mata nativa 1 ; e MN-2: mata nativa 2 .

Means followed by the same letter, capitals in the columns and small in the lines, are not significantly different by the Tukey test at 5\% level. AGP: agrosilvopasture; SILV: silvopasture; TR98: traditionally cultivated in 1998; TR02: traditionally cultivated in 2002; CI: intensive cropping; $M N-1$ : native forest $1 ; M N-2$ : native forest 2. 
Tabela 6 - Médias dos teores de carbono orgânico total nos diferentes tratamentos agroflorestal, e convencional e em áreas nativas, nas profundidades de 0-6, 6$12,12-20$ e $20-40 \mathrm{~cm}$

Table 6-Total organic carbon contents from agroforestry, conventional and native forest sites at the 0-6, 612, $12-20$ and 20-40 cm layers

\begin{tabular}{lllll}
\hline Tratamentos & \multicolumn{4}{c}{ Camada, cm } \\
\cline { 2 - 5 } & $0-6$ & $6-12$ & $12-20$ & $20-40$ \\
\hline \multicolumn{5}{c}{ dag dm $^{-3}$} \\
\hline AGP & 2,23 & 1,44 & 1,15 & 0,88 \\
SILV & 3,48 & 1,72 & 1,47 & 1,28 \\
TR98 & 2,35 & 1,82 & 1,35 & 1,12 \\
TR02 & 3,44 & 2,31 & 1,67 & 1,26 \\
CI & 2,16 & 1,31 & 1,07 & 0,74 \\
MN-1 & 3,62 & 2,07 & 1,77 & 1,26 \\
MN-2 & 2,72 & 1,49 & 1,05 & 0,67 \\
\hline
\end{tabular}

AGP: agrossilvipastoril; SILV: silvipastoril; TR98: tradicional de 1998; TR02: tradicional de 2002; CI: cultivo intensivo; MN-1: mata nativa $1 ; \mathrm{MN}-2$ : mata nativa 2 .

AGP: agrosilvopasture; SILV: silvopasture; TR98: traditionally cultivated in 1998; TRO2: traditionally cultivated in 2002; CI: intensive cropping; $M N-1$ : native forestry 1; MN-2: native forestry 2.

Diferentes sistemas de manejo afetam os teores de COT do solo pela modificação na taxa de decomposição da matéria orgânica e por meio da alteração do aporte anual de resíduos orgânicos (BAYER e MIELNICZUCK, 1997). Os tratamentos AGP e SILV, com aportes líquidos de MS de aproximadamente 4,0 e 4,48 $\mathrm{Mg} \mathrm{ha}^{-1} \mathrm{ano}^{-1}$, respectivamente, apresentaram taxas anuais de aporte superiores ao observado na MN-1 (3,7 Mg ha-1 ano $\left.^{-1}\right)$. Tais tratamentos mostramse mais eficientes em aportar matéria orgânica ao solo, significando também maior eficiência na ciclagem de nutrientes. Já os tratamentos do modelo tradicional TR98 e TR02 tiveram um aporte médio líquido anual de $1,75 \mathrm{Mg} \mathrm{ha}^{-1}$, enquanto o tratamento CI apresentou $1,36 \mathrm{Mg} \mathrm{ha}^{-1} \mathrm{ano}^{-1}$, o que implica diminuição da ciclagem de nutrientes nesses sistemas.

A maior ciclagem de nutrientes no tratamento AGP é, provavelmente, a responsável pela manutenção de altos índices de produtividade do milho. De acordo com Araújo Filho (2003), esse tratamento apresentou produtividade média de $1.376,0 \mathrm{~kg} \mathrm{ha}^{-1} \mathrm{ano}^{-1}$ de milho, distribuída uniformemente durante os cinco anos de cultivo. Entretanto, o tratamento CI obteve produtividade média de apenas $542 \mathrm{~kg} \mathrm{ha}^{-1}$ ano $^{-1}$, observando-se uma queda gradativa na produtividade, que se iniciou no primeiro ano (1998), obtendo-se $788 \mathrm{~kg} \mathrm{ha}^{-1}$, e alcançando $281 \mathrm{~kg} \mathrm{ha}^{-1}$ em 2001.

R. Árvore, Viçosa-MG, v.30, n.5, p.837-848, 2006
Apesar de os tratamentos modificarem o aporte anual de resíduos orgânicos, quando relacionados ao tratamento MN-1 nenhum deles apresentou déficit. Isso significa que as perdas de COT foram geradas principalmente por alterações na taxa de decomposição da matéria orgânica do solo (MOS). Nos tratamentos AGP e CI, a diminuição nos teores de COT (Tabela 6) deveu-se, principalmente, à oxidação da matéria orgânica gerada pelo revolvimento do solo. No AGP, a erosão teve participação secundária, pois as quantidades de solo e água perdidas, obtidas por Maia (2004), estão muito abaixo dos valores encontrados na literatura. Já no tratamento CI, a análise dos resultados de perda de solo e água (MAIA, 2004), juntamente com os de estabilidade de agregados (Tabela 7), indicaram que nesse tratamento a erosão hídrica foi também importante na diminuição do teor de COT.

O tratamento TR98 reduziu os teores de COT em 54,4 e $13,7 \%$, nas camadas de $0-6$ e $6-12 \mathrm{~cm}$, respectivamente (Tabela 6 ). No entanto, o TR02 apresentou, em algumas situações, teores de COT superiores àqueles obtidos no tratamento $\mathrm{MN}-1$, o que provavelmente ocorreu devido ao fato de esse sistema ainda estar sob efeito da vegetação nativa que o antecedia, ou seja, apenas um cultivo não foi suficiente para provocar alterações na MOS dessa área.

Os resultados do tratamento SILV ressaltam o efeito do revolvimento do solo sobre a MOS. Sendo o menos intensivo entre os avaliados, esse sistema praticamente não apresentou redução nos teores de COT.

\subsection{Estabilidade de agregados}

A distribuição porcentual dos agregados estáveis em água nas diferentes classes de diâmetro, tratamentos e profundidades estudados encontra-se na Tabela 7. Para a comparação dos efeitos dos tratamentos, tomouse a área de mata nativa $\mathrm{MN}-1$ como condição original de agregação do solo.

Os maiores efeitos dos tratamentos manifestaramse nas classes C1(4,76-2,0 mm) e C5 (<0,25 mm). Nas demais, observou-se maior homogeneidade, tanto entre os tratamentos como entre as profundidades. Notou-se também que a $\mathrm{C} 1$ predominou na camada de $0-6 \mathrm{~cm}$ e diminuiu gradualmente com a profundidade até ser superada pela C5 a partir da camada de 12$20 \mathrm{~cm}$ (Tabela 7). 
Tabela 7 - Distribuição dos porcentuais médios dos agregados estáveis em água nas diferentes classes de diâmetro nos diferentes tratamentos agroflorestal e convencional e em áreas nativas, nas profundidades de 0-6, 6-12, 12-20 e 20-40 cm

Table 7-Percentage of water stable aggregates from agroforestry, conventional and native forest sites at the 0-6, 6-12, $12-20$ and $20-40 \mathrm{~cm}$ layers

\begin{tabular}{|c|c|c|c|c|c|}
\hline \multirow{3}{*}{ Tratamentos } & \multicolumn{5}{|c|}{ \% de agregados estáveis em água } \\
\hline & \multicolumn{5}{|c|}{ Classes de diâmetro, $\mathrm{mm}$} \\
\hline & $4,76-2,0(\mathrm{C} 1)$ & $2,0-1,0(\mathrm{C} 2)$ & $1,0-0,5(\mathrm{C} 3)$ & $0,5-0,25(\mathrm{C} 4)$ & $<0,25(\mathrm{C} 5)$ \\
\hline AGP & $49,03 \mathrm{C}$ & $10,13 \mathrm{~A}$ & $\begin{array}{l}0-6 \mathrm{~cm} \\
7,28 \mathrm{~A}\end{array}$ & $6,64 \mathrm{~A}$ & $26,89 \mathrm{~A}$ \\
\hline SILV & $87,55 \mathrm{~A}$ & $2,84 \mathrm{~B}$ & $0,42 \mathrm{D}$ & $0,405 \mathrm{D}$ & $8,77 \mathrm{BC}$ \\
\hline TR98 & $68,31 \mathrm{~B}$ & $12,25 \mathrm{~A}$ & $3,00 \mathrm{BC}$ & $2,34 \mathrm{BC}$ & $14,08 \mathrm{BC}$ \\
\hline TR02 & $80,45 \mathrm{AB}$ & 9,92 A & $2,06 \mathrm{BCD}$ & $1,38 \mathrm{BCD}$ & $6,18 \mathrm{C}$ \\
\hline CI & $70,95 \mathrm{~B}$ & $10,01 \mathrm{~A}$ & $2,27 \mathrm{BCD}$ & $1,56 \mathrm{BCD}$ & $15,18 \mathrm{~B}$ \\
\hline MN-1 & $66,05 \mathrm{~B}$ & $13,74 \mathrm{~A}$ & $3,66 \mathrm{~B}$ & $2,55 \mathrm{~B}$ & $13,98 \mathrm{BC}$ \\
\hline \multirow[t]{2}{*}{$\mathrm{MN}-2$} & $76,81 \mathrm{AB}$ & $10,71 \mathrm{~A}$ & $1,14 \mathrm{CD}$ & $0,76 \mathrm{CD}$ & $10,56 \mathrm{BC}$ \\
\hline & & & 6-12 cm & & \\
\hline AGP & $18,11 \mathrm{C}$ & $10,28 \mathrm{BC}$ & $12,70 \mathrm{AB}$ & $17,35 \mathrm{~A}$ & $41,53 \mathrm{~A}$ \\
\hline SILV & $56,90 \mathrm{~A}$ & $7,87 \mathrm{C}$ & $3,89 \mathrm{C}$ & $1,47 \mathrm{~B}$ & $29,85 \mathrm{AB}$ \\
\hline TR98 & $39,09 \mathrm{ABC}$ & $19,31 \mathrm{~A}$ & $8,80 \mathrm{ABC}$ & $4,89 \mathrm{~B}$ & $27,88 \mathrm{AB}$ \\
\hline TR02 & $58,29 \mathrm{~A}$ & $17,54 \mathrm{~A}$ & $7,42 \mathrm{BC}$ & $3,40 \mathrm{~B}$ & $13,33 \mathrm{~B}$ \\
\hline CI & $36,01 \mathrm{ABC}$ & $16,22 \mathrm{AB}$ & $7,94 \mathrm{BC}$ & $5,32 \mathrm{~B}$ & $34,5 \mathrm{AB}$ \\
\hline MN-1 & $21,18 \mathrm{BC}$ & $18,50 \mathrm{~A}$ & $15,44 \mathrm{~A}$ & $8,31 \mathrm{AB}$ & $36,55 \mathrm{AB}$ \\
\hline \multirow[t]{2}{*}{$\mathrm{MN}-2$} & $48,10 \mathrm{AB}$ & $16,99 \mathrm{~A}$ & $4,68 \mathrm{C}$ & $2,10 \mathrm{~B}$ & $28,11 \mathrm{AB}$ \\
\hline & & & $12-20 \mathrm{~cm}$ & & \\
\hline AGP & $19,84 \mathrm{~B}$ & $16,04 \mathrm{AB}$ & $14,28 \mathrm{AB}$ & $12,34 \mathrm{~A}$ & $\begin{array}{l}37,50 \\
34,32\end{array}$ \\
\hline SILV & $49,77 \mathrm{~A}$ & 7,95 B & $4,42 \mathrm{D}$ & $3,54 \mathrm{~B}$ & 34,32 \\
\hline TR98 & $35,14 \mathrm{AB}$ & $23,26 \mathrm{~A}$ & $10,11 \mathrm{BC}$ & $6,90 \mathrm{AB}$ & 24,59 \\
\hline TR02 & $37,16 \mathrm{AB}$ & $17,77 \mathrm{~A}$ & $10,38 \mathrm{BC}$ & $10,00 \mathrm{AB}$ & 24,69 \\
\hline CI & $24,25 \mathrm{AB}$ & $15,82 \mathrm{AB}$ & $11,11 \mathrm{BC}$ & $10,21 \mathrm{AB}$ & 38,61 \\
\hline MN-1 & $18,82 \mathrm{~B}$ & $22,43 \mathrm{~A}$ & $16,55 \mathrm{~A}$ & $12,54 \mathrm{~A}$ & 29,66 \\
\hline \multirow[t]{2}{*}{$\mathrm{MN}-2$} & $41,30 \mathrm{AB}$ & $18,10 \mathrm{~A}$ & $6,36 \mathrm{CD}$ & $4,32 \mathrm{~B}$ & 29,92 \\
\hline & & & $20-40 \mathrm{~cm}$ & & \\
\hline AGP & $16,36 \mathrm{AB}$ & $14,60 \mathrm{AB}$ & $14,60 \mathrm{AB}$ & $12,50 \mathrm{AB}$ & $41,87 \mathrm{AB}$ \\
\hline SILV & $28,46 \mathrm{AB}$ & $7,83 \mathrm{~B}$ & $8,66 \mathrm{C}$ & $5,42 \mathrm{C}$ & $49,61 \mathrm{~A}$ \\
\hline TR98 & $13,41 \mathrm{AB}$ & $21,50 \mathrm{~A}$ & $14,29 \mathrm{ABC}$ & $8,09 \mathrm{BC}$ & $42,66 \mathrm{AB}$ \\
\hline TR02 & 29,07 AB & $24,05 \mathrm{~A}$ & $15,19 \mathrm{~A}$ & $8,05 \mathrm{BC}$ & $23,62 \mathrm{~B}$ \\
\hline CI & $17,27 \mathrm{AB}$ & $14,20 \mathrm{AB}$ & $14,81 \mathrm{AB}$ & $10,96 \mathrm{AB}$ & $42,74 \mathrm{AB}$ \\
\hline MN-1 & $9,85 \quad$ B & $19,00 \mathrm{~A}$ & $19,93 \mathrm{~A}$ & $12,78 \mathrm{~A}$ & $38,42 \mathrm{AB}$ \\
\hline $\mathrm{MN}-2$ & $30,10 \mathrm{~A}$ & $15,11 \mathrm{AB}$ & $9,46 \mathrm{BC}$ & $5,27 \mathrm{C}$ & $40,05 \mathrm{AB}$ \\
\hline
\end{tabular}

Médias seguidas de mesmas letras maiúsculas nas colunas e minúsculas nas linhas não diferem entre si, pelo teste de Tukey a 5\%. AGP: agrossilvipastoril; SILV: silvipastoril; TR98: tradicional de 1998; TR02: tradicional de 2002; CI: cultivo intensivo; MN-1: mata nativa 1 ; e MN-2: mata nativa 2.

Means followed by the same letter, capitals in the columns and small in the lines, are not significantly different by the Tukey test at $5 \%$ level. AGP: agrosilvopasture; SILV: silvopasture; TR98: traditionally cultivated in 1998; TRO2: traditionally cultivated in 2002; CI: intensive cropping; $M N-1$ : native forest $1 ; M N-2$ : native forest 2.

A diminuição dos macroagregados em profundidade parece estar fortemente relacionada ao teor do COT (OLIVEIRA et al., 1983; PALADINI e MIELNICZUK, 1991; HAYNES, 1999; PALMEIRAet al., 1999). No presente estudo, a diminuição da $\mathrm{C} 1$ é concomitante à redução do teor de COT (Tabela 6). O coeficiente de correlação entre as duas variáveis foi de 0,70 .

Na camada de 0-6 cm, o tratamento AGP apresentou o menor porcentual de agregados $(49,03 \%)$ na $\mathrm{C} 1 \mathrm{e}$ os maiores nas classes C3 (7,28\%), C4 (6,64\%) e C5
$(26,89 \%)$, diferindo dos demais tratamentos. O maior porcentual de agregados estáveis na $\mathrm{C} 1$ foi observado no tratamento SILV $(87,5 \%)$ igual a TR02 $(80,45 \%)$ e MN-2 (76,8\%), diferindo dos demais, inclusive de MN$1(66,0 \%)$. Nas demais camadas ocorreram a diminuição gradual da $\mathrm{C} 1$ e o aumento da $\mathrm{C} 5$, no entanto os resultados seguiram o observado na camada de $0-6 \mathrm{~cm}$, com o tratamento AGP apresentando sempre menores porcentuais de macroagregados e o SILV, os maiores (Tabela 7). 
O solo sob o tratamento AGP, em função dos cinco anos de cultivo, tendeu a perder a estrutura original pelo fracionamento dos agregados maiores em unidades menores. De acordo com Tisdall e Oades (1982), nos solos cultivados os agregados são expostos freqüentemente à desagregação física, seja pelo rápido umedecimento e pelo impacto da gota de chuva, seja pelo cisalhamento causado por implementos agrícolas.

Os resultados obtidos no tratamento SILV confirmam os efeitos do revolvimento sobre a estrutura do solo. Observou-se aumento significativo dos macroagregados (C1) em relação ao tratamento-referência MN-1, atribuído primeiramente ao não-revolvimento do solo e, posteriormente, ao aumento da cobertura do solo promovida pela vegetação herbácea. O raleamento do estrato arbóreo-arbustivo realizado favoreceu o desenvolvimento do estrato herbáceo em função das melhores condições de luminosidade. Observou-se que o tratamento SILV obteve, em média, $60,5 \%$ de cobertura do solo, enquanto que MN-1 apenas 29,5\% (MAIA, 2004).

A ação das plantas na formação e estabilização dos agregados tem sido amplamente ressaltada (CAMPOS et al., 1995; SILVA e MIELNICZUK, 1997a; SILVA e MIELNICZUK, 1997b). As raízes produzem agregação estável mediante o suprimento de resíduos orgânicos para a decomposição, exsudações de substâncias orgânicas, envolvimento físico de microagregados e reorientação e aproximação de partículas e microagregados por dessecamentos localizados (SILVA e MIELNICZUK, 1997b).

Apenas os resultados encontrados no tratamento CI foram contrários ao consenso de que quanto mais intensivo for o sistema, maior será seu impacto sobre as condições físicas do solo (DA ROS et al., 1997). Este tratamento foi cultivado convencionalmente por cinco anos (1997-2001) e teve seus teores de COT substancialmente reduzidos (Tabela 6). No entanto, apresentou condição de estabilidade de agregados similar à área de referência MN-1. No momento da coleta das amostras de solo, essa área estava em pousio há aproximadamente cinco meses, observando-se a predominância de gramíneas de crescimento espontâneo. Entretanto, é pouco provável que esse curto período de pousio e a presença das gramíneas tenham sido capazes de recuperar a estrutura do solo. Outra explicação seria a estabilização dos agregados a partir da junção de partículas minerais (principalmente silte e argila). Segundo Harris et al. (1966), alguns processos conduzem as partículas de argila a uma orientação preferencial pelas partículas vizinhas, envolvendo a atração de cátions dissociados aos minerais de argila carregados negativamente. Tal quadro pode ter sido intensificado ao considerar-se o aumento natural dos teores de argila nas camadas subsuperficiais expostas pela erosão hídrica. Os Luvissolos são a ordem de solos predominantes, sendo caracterizados por um horizonte B textural com argila ativa, o que torna os fenômenos de coesão e adesão mais intensos, favorecendo a formação de agregados mais estáveis, com estruturas em blocos moderada a fortemente desenvolvidas, ou subangulares prismática, composta de blocos angulares ou subangulares (EMBRAPA, 1999).

\section{CONCLUSÕES}

Os atributos químicos do solo, como bases trocáveis, CTC, pH e acidez potencial no tratamento SILV, variaram em função dos teores de argila. Nos tratamentos TR98 e CI houve redução nesses atributos devido ao maior revolvimento do solo, enquanto que, o tratamento AGP mostrou-se eficiente na ciclagem de nutrientes, apesar da redução no teor de COT.

O estado de agregação do solo foi reduzido no tratamento AGP devido ao maior revolvimento e à conseqüente diminuição no teor de COT, enquanto no tratamento SILV, em que não houve revolvimento do solo, ocorreu incremento na estabilidade dos macroagregados $(>2,0 \mathrm{~mm})$.

O tratamento AGP poderá proporcionar erosão do solo, refletindo em drástica redução de sua qualidade. Os resultados permitiram recomendar o tratamento silvipastoril (SILV) para a manutenção da qualidade do solo e a produção de alimentos na região do semiárido cearense.

\section{AGRADECIMENTOS}

Este trabalho foi financiado pelo Programa de Cooperação Acadêmica (PROCAD) da CAPES, que envolveu as Universidades Federais do Ceará (UFC) e Viçosa (UFV). Agradecemos ao Sr. Braz Júlio (UFV) pelo auxilio nas análises de solo e ao Centro Nacional de Pesquisas de Caprinos - CNPC / EMBRAPA por viabilizar o trabalho na Fazenda Crioula. 


\section{REFERÊNCIAS BIBLIOGRÁFICAS}

ARAÚJO FILHO, J.A. Caatinga: agroecologia versus desertificação. Ciência Hoje, v. 30, n. 180, p. 44-45, 2002.

ARAÚJO FILHO, J.A. Sistemas de produção sustentáveis para a região da caatinga.

Relatório Final de Projeto. Sobral: CNPC/ EMBRAPA, 2003. 14 p.

ARAÚJO, G.G.L. et al. Opções no uso de forrageiras arbustivo-arbóreas na alimentação animal no semiárido do nordeste. In: CARVALHO, M.M. et al. Sistemas agroflorestais pecuários: opções de sustentabilidade para áreas tropicais e subtropicais. Juiz de Fora: 2001. p. 111-137.

BAYER, C.; MIELNICZUK, J. Características químicas do solo afetadas por métodos de preparo e sistemas de cultura. Revista Brasileira de Ciência do Solo, v. 21, p. 105-112, 1997.

BREMAN, H.; KESSLER, J.J. The potential benefits of agroforetry in the Sahel and other semi - arid regions. European Journal of Agronomy, v. 7, p. 25-33, 1997.

CAMPOS, B.C. et al. Estabilidade estrutural de um Latossolo Vermelho-Escuro distrófico após sete anos de rotação de culturas e sistemas de manejo de solo. R. Bras. Ci. Solo, v. 19, p. 121-126, 1995.

DA ROS, C.O. et al. Manejo do solo a partir de campo nativo: efeito sobre a forma e estabilidade da estrutura ao final de cinco anos. Revista Brasileira de Ciência do Solo, v. 21, p. 241-247, 1997.

DAY, P.R. Particle fractionation and particle size analysis. In: BLACK,C.A. Methods of soil analysis. Madison: American Society of Agronomy, 1965. p. 545-567.

EMPRESA BRASILEIRA DE PESQUISA AGROPECUÁRIA - EMBRAPA. Centro Nacional de Pesquisa de Solos. Manual de métodos de análise de solo. 2.ed. Rio de Janeiro, 1997. 212p.

EMPRESA BRASILEIRA DE PESQUISA AGROPECUÁRIA - EMBRAPA. Centro Nacional de Pesquisa de Solos. Sistema brasileiro de classificação de solos. Brasília: EMBRAPA Produção de Informação, 1999. 412 p.
FRANZEL, S. et al. Assessing the adoption potential of agroforestry practices in sub-Saharan Africa.

Agricultural Systems, v. 69, p. 37-62, 2001.

HARRIS, R.F.; CHESTERS, G.; ALLEN, O.N. Dynamics of soil aggregation. Advances in Agronomy, v. 18, p. 107-169, 1966.

HAYNES, R.J. Labile organic matter fractions and aggregate stability under short-term, grass-based leys. Soil Biology Biochemistry, v. 31, p. 1821-1830, 1999.

IZAC, A.M.N.; SANCHEZ, P.A. Towards a natural resource management paradigm for international agriculture: the example of agroforestry research. Agricultural Systems, v. 69, p. 5-25, 2001.

KLEPKER, D.; ANGHINONI, I. Características físicas e químicas do solo afetadas por métodos de preparo e modos de adubação. Revista Brasileira de Ciência do Solo, v. 19, p. 395-401, 1995.

LUZ, L.R.Q.P.; SANTOS, M.C.D.; MERMUT, A.R. Pedogênese em uma topossequência do semi-árido de Pernambuco. Revista Brasileira de Ciência do Solo, v. 16, p. 95-102, 1992.

MAIA, S.M.F. Compartimentos da matéria orgânica e perdas de solo e água em sistemas agroflorestais e convencional no trópico semi - árido cearense. 2004. 98 f. Dissertação (Mestrado em Solos e Nutrição de Plantas) - Universidade Federal do Ceará, Fortaleza, 2004.

MELLO, F.A.F. et al. Fertilidade do solo. 4. ed. São Paulo: Nobel, 1983. 400 p.

MENDONZA, H.N.S. et al. Propriedades químicas e biológicas de solo de tabuleiro cultivado com cana-de-açúcar com e sem queima da palhada. Revista Brasileira de Ciência do Solo, v. 24, p. 201-207, 2000.

NAIR, P.K.R. An introduction to agroforestry. Dordrecht: Kluwer Academic Publishers, 1993. 499p.

NAIR, P.K.R. et al. Nutrient cycling in tropical agroforestry systems: myths and science. In: BUCK, L.E.; LASSOIE, J.P.; FERNANDES, E.C.M. Agroforestry in sustainable agricultural systems. Boca Raton: 1999, p. 1-31.

R. Árvore, Viçosa-MG, v.30, n.5, p.837-848, 2006 
OLIVEIRA, M.; CURI, N.; FREIRE, J.C. Influência do cultivo na agregação de um Podzólico Vermelho- Amarelo textura média argilosa da região de Lavras (MG). Revista Brasileira de Ciência do Solo, v. 7, p. 317-322, 1983.

PALADÍNI, F.L.S.; MIELNICZUK, J. Distribuição de tamanho de agregados de um solo Podzólico Vermelho- Escuro afetado por sistema de culturas. Revista Brasileira de Ciência do Solo, v. 15, p. 135-140, 1991.

PALMEIRA, P.R.T. et al. Agregação de um Planossolo submetido a diferentes sistemas de cultivo. Revista Brasileira de Ciência do Solo, v. 23, p. 189-195, 1999.

ROOSE, E.; NDAYIZIGIYE, F. Agroforestry, water and soil fertility management to fight erosion in tropical mountains of Rwanda. Soil

Technology, v. 11, p. 109-119, 1997.

SÁNCHEZ, M.D. Panorama dos sistemas agroflorestais pecuários na América Latina. In: CARVALHO, M.M.; ALVIM, M.J.; CARNEIRO, J.C. Sistemas agroflorestais pecuários: opções de sustentabilidade para áreas tropicais e subtropicais. Juiz de Fora: 2001. p. 9-17.

SCHROTH, GOTZ et al. Conversion os secondary Forest into agroforestry and monoculture plantations in Amazônia: consequences for biomass, litter and soil carbon stocks after 7 years.

Forestry Ecology and Managenment, v. 163, p. 131-150, 2002.
SILVA, I.F.; MIELNICZUK, J. Avaliação do estado de agregação do solo afetado pelo uso agrícola. Revista Brasileira de Ciência do Solo, v. 21, p. 313-319, 1997a.

SILVA, I.F.; MIELNICZUK, J. Ação do sistema radicular de plantas na formação e estabilização de agregados do solo. Revista Brasileira de Ciência do Solo, v. 21, p. 113-117, 1997 b.

SOLOMON, D.; LEHMANN, J.; ZECH, W. Land use effects on soil organic matter properties of chromic luvisols in semi-arid northern Tanzania: carbon, nitrogen, lignin and carbohydrates. Agriculture Ecosystems and Environment, v. 78, p. 203-213, 2000.

TAN, K.H. Principles of soil chemistry. 3 ed. New York: Marcel Dekker, 1998. 267p.

THEODORO, V.C.A. et al. Alterações químicas em solo submetido a diferentes formas de manejo do cafeeiro. Revista Brasileira de Ciência do Solo, v. 27, p. 1039-1047, 2003.

TISDALL, J.M.; OADES, J.M. Organic matter and water-stable aggregates in soils. Journal of Soil Science, v. 33, p. 141-163, 1982.

YEOMANS, J.C.; BREMNER, J.M. A rapid and precise method for routine determimation of organic carbon in soil. Communications in Soil Science Plant Analysis, v. 19, p. 1467-1476, 1988. 